\title{
Percutaneous Coronary Intervention for High Risk Non-ST Elevation Myocardial Infarction or Unstable Angina
}

National Cancer Institute

\section{Source}

National Cancer Institute. Percutaneous Coronary Intervention for High Risk Non-ST

Elevation Myocardial Infarction or Unstable Angina. NCI Thesaurus. Code C99999.

Includes patients with unstable ang ina or Non-ST EMI who have high risk features for short-term risk of death or nonfatal MI. High risk features includes at least one of the following: history of accelerating tempo of ischemic symptoms in preceding 48 hours; character of pain - prolonged ongoing (greater than 20 minutes) rest pain; clincal findings - pulmonary edema, most likely due to ischemia or new or worsening mitral regurgitation murmur; or S3 or new worsening rales or Hypotension, bradycardia, tachycardia or age greater than 75 years; ECG - ang ina at rest with transient ST -segment chang es greater than $0.5 \mathrm{~mm}$ or bundle-branch block, new or presumed new or sustained ventricular tachycardia; cardiac markers - NSTEMI patients with elevated cardiac TnT, Tnl, or CKMB. (ACC) 